\title{
Evaluation of safety and efficacy of tivantinib in the treatment of inoperable or recurrent non-small-cell lung cancer
}

This article was published in the following Dove Press journal:

Cancer Management and Research

16 January 2013

Number of times this article has been viewed

\author{
Massimo Broggini' \\ Marina Chiara Garassino² \\ Giovanna Damia' \\ 'Laboratory of Molecular \\ Pharmacology, Istituto di Ricerche \\ Farmacologiche "Mario Negri", \\ 2Division of Medical Oncology, \\ Fondazione IRCCS National Cancer \\ Institute, Milan, Italy
}

\begin{abstract}
Tivantinib is a selective, oral, non-ATP-competitive, small molecule inhibitor of the c-Met receptor, tyrosine kinase, which is implicated at different levels of tumor cell migration, invasion, proliferation, and metastasis. Tivantinib has shown antitumor activity in various human tumor cell lines and in xenograft models of human cancers, including non-small-cell lung cancer. Few therapeutic options are available at present for advanced non-small-cell lung cancer, so there is a pressing need for new therapeutic strategies to improve response and survival. Amplification of Met has been reported in more than $20 \%$ of lung tumors that have acquired resistance to epidermal growth factor receptor inhibitors, implying that treatment of these tumors with a c-Met inhibitor should overcome resistance. Tivantinib has shown interesting and promising results in advanced non-small-cell lung cancer and appears to be well tolerated, either alone or in combination with other drugs. An interesting additional feature is the ability of the drug to delay development of new metastasis, in agreement with the proposed role of Met in this particular setting.
\end{abstract}

Keywords: non-small-cell lung cancer, Met inhibitors, tivantinib, biomarkers

\section{Management issues in non-small-cell lung cancer}

Lung cancer is the leading cause of cancer-related deaths in western countries and is one of the major health problems worldwide, with more than 1.5 million new cases per year. ${ }^{1,2}$ Non-small-cell lung cancer (NSCLC) accounts for more than $80 \%$ of lung tumors. The standard first-line treatment for advanced NSCLC is platinum-based doublet chemotherapy, but median survival is still less than one year. ${ }^{3}$

Increasing knowledge of the molecular pathophysiology of NSCLC has led to the development of new targeted therapies which have somewhat improved survival. The presence of the mutated epidermal growth factor receptor (EGFR) gene in NSCLC is associated with responsiveness to EGFR tyrosine kinase inhibitors (erlotinib, gefitinib) and these are now approved as first-line treatments for patients with EGFR mutation. ${ }^{4-7}$ Encouraging results have been obtained with afatinib, an irreversible EGFR tyrosine kinase inhibitor. ${ }^{8}$ Similarly, patients with tumors harboring anaplastic lymphoma kinase EML4 translocation are sensitive to anaplastic lymphoma kinase inhibitors such as crizotinib, which has demonstrated superiority to chemotherapy in terms of progression-free survival. ${ }^{9}$

However, these substantial improvements in the management of patients with NSCLC are beneficial to only a small percentage of patients (approximately $10 \%$ and $4 \%$ of total patients with NSCLC of Caucasian origin have alterations in EGFR and anaplastic lymphoma kinase, respectively). The percentage of patients with NSCLC presenting with alterations in EGFR is higher among Asians than Caucasians, and the majority of the remaining cases do not benefit, at best, from targeted therapies. In fact,
Correspondence: Massimo Broggini Laboratory of Molecular Pharmacology Istituto di Ricerche Farmacologiche “Mario Negri”, via La Masa 19,

Milan 20156, Italy

Tel +3902 390। 4585

Fax +3902 390I 4734

Email massimo.broggini@marionegri.it 
there is recent evidence that anti-EGFR therapy may be inferior to standard chemotherapy as second line in patients with NSCLC and wild-type EGFR. ${ }^{10}$

In addition, it has to be pointed out that patients initially responding to targeted therapies will eventually develop resistance, hence limiting the potential effectiveness of these treatments. Mechanisms of resistance to targeted therapies include appearance of secondary mutations (both in patients with EGFR and anaplastic lymphoma kinase EML) which are no longer sensitive to selective inhibitors. ${ }^{11-14}$ Another mechanism of resistance is represented by amplification of the Met gene. ${ }^{15,16}$ Therefore, new therapeutic strategies are urgently needed for advanced NSCLC.

A study by the National Cancer Institute's Lung Cancer Mutation Consortium indicated that more than $60 \%$ of patients with NSCLC harbor mutations which could affect new therapeutic modalities. ${ }^{17}$ Met is among the candidates, in view of the evidence discussed above that a certain percentage of resistant tumors show amplification of the Met gene and that Met is strongly implicated in metastasis. ${ }^{18}$ Preclinical evidence further indicates that Met is a good candidate target in NSCLC. Met expression is found in virtually all NSCLC samples analyzed and in approximately $90 \%$ of NSCLC cell lines. Constitutive activation of Met has also been observed in clinical samples and cell lines (Figure 1). ${ }^{18-22}$ Two compounds interfering with the Met signaling pathway are at present in advanced clinical studies in NSCLC, ie, a monoclonal antibody directed against Met (onartuzumab) and tivantinib, a small molecule which is a non-ATP-competitive inhibitor of Met. ${ }^{21,23}$
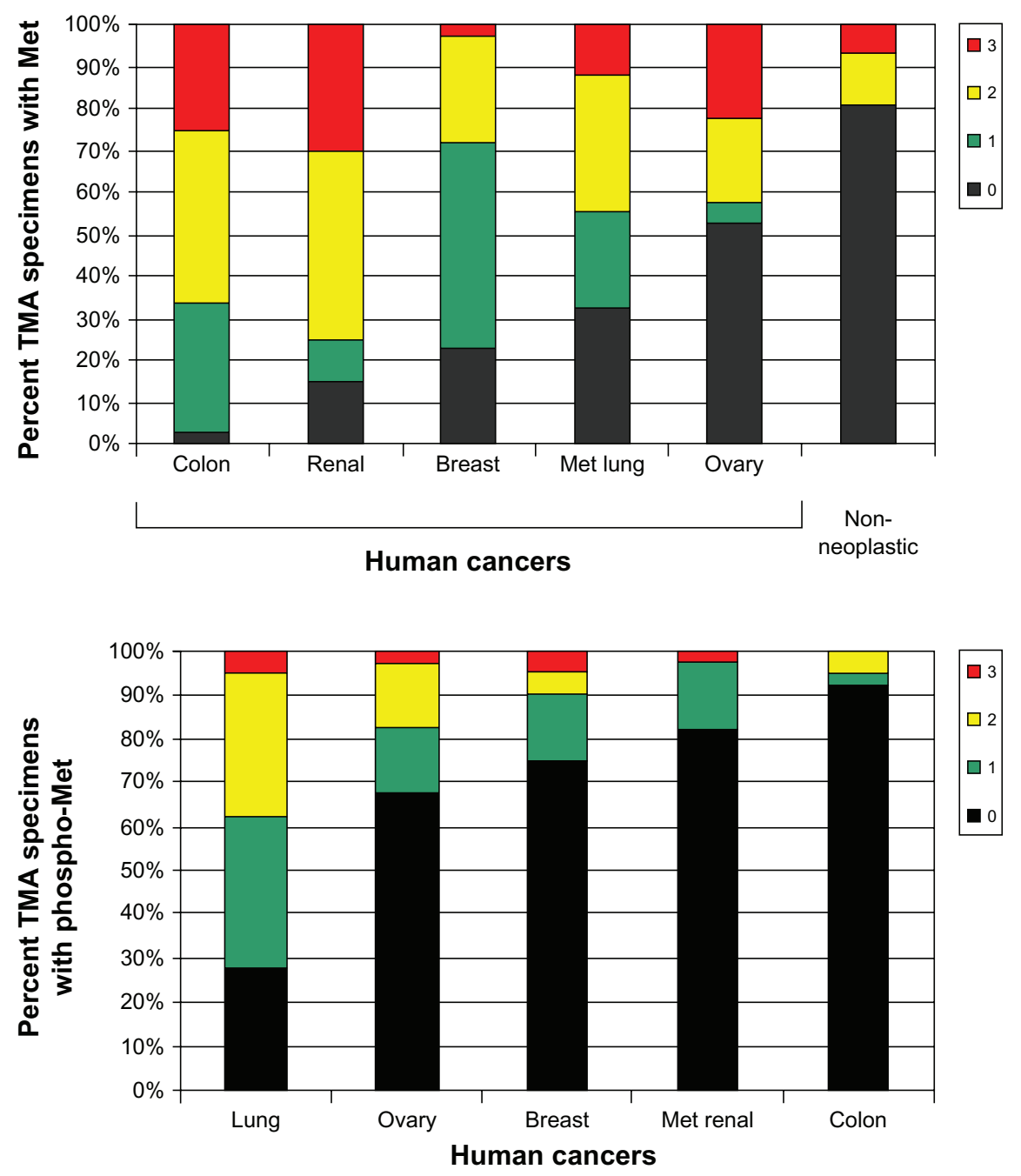

Figure I Expression (upper panel determined using a total anti-Met antibody) and activation (lower panel, determined using a phosphospecific Met antibody) of Met in non-small-cell lung cancer and other human tumors.

Notes: The different colors and the corresponding numbers refer to negative ( 0 , black), weak (I, green), moderate (2, yellow), or strong ( 3 , red) staining.

Copyright (C) 2008. John Wiley and Sons. Adapted from Ma PC, Tretiakova MS, MacKinnon AC, et al. Expression and mutational analysis of MET in human solid cancers. Genes Chromosomes Cancer. 2008;47( I2): 1025-1037. ${ }^{19}$

Abbreviation: TMA, tissue microarray. 


\section{Pharmacology, mode of action, pharmacokinetics of tivantinib}

Tivantinib is a small molecule, non-ATP-competitive inhibitor of Met (see chemical structure in Figure 2). It has relatively high specificity for Met, with no appreciable inhibition of the activity of 229 other kinases tested. ${ }^{21}$ Tivantinib inhibits Met autoactivation by selectively targeting the inactive form of the kinase. ${ }^{24}$ This results in inhibition of Met signaling pathways downstream.

Tivantinib has shown activity in several human cancer cell lines grown in vitro. In NSCLC cells, tivantinib showed cytotoxic activity, with concentrations inhibiting cell growth by $50 \%\left(\mathrm{IC}_{50}\right)$ below $1 \mu \mathrm{M}$ in cells expressing Met. ${ }^{21}$ In one NSCLC cell line, with confirmed lack of Met expression, the compound had no significant activity, with an $\mathrm{IC}_{50}>30 \mu \mathrm{M}$. The in vitro cytotoxic activity of tivantinib is associated with a decrease in Met phosphorylation and reduced Met signaling downstream. Tivantinib inhibited tumor growth in vivo in immunodeficient mice implanted with human tumors. Given as repeated oral doses, the compound showed antitumor activity at nontoxic doses. ${ }^{21}$

$K-R A S$ mutant NSCLCs are associated with a poor response to classical chemotherapy as well as targeted therapy. ${ }^{25-27}$ Tivantinib showed activity independently from K-RAS status. In particular, isogenic NSCLC cells only differing in expression of mutant or wild-type K-RAS showed the same response to tivantinib in vitro ${ }^{21}$ (Caiola et al, manuscript in preparation). Finally, in preclinical tests, tivantinib has shown promising antimetastatic effects, inhibiting bone metastasis formation in vivo even at doses with no direct cytotoxic activity. ${ }^{28}$

The compound is oxidatively metabolized by CYP2C19 and CYP3A4 isozymes of the cytochrome p450 family. ${ }^{29}$ In preclinical studies, oral bioavailability was found to be higher than $20 \%$ in mice and dogs. ${ }^{29}$ Pharmacokinetic

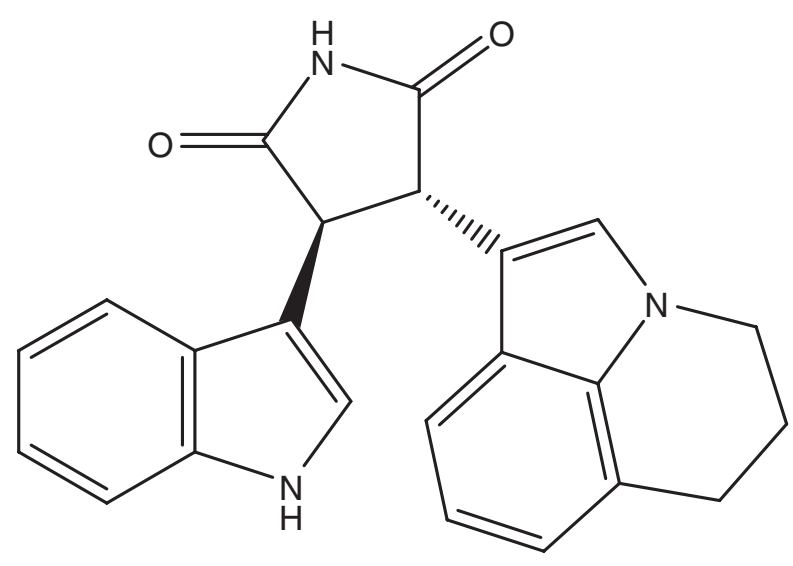

Figure 2 Chemical structure of tivantinib. analysis in Phase I studies indicated linearity, with a halflife ranging from 3 to 6 hours, up to a dose of $400 \mathrm{mg}$ twice a day, where clearance saturation might occur. ${ }^{30}$ In Phase I studies where the drug was given as monotherapy to patients with NSCLC, tivantinib achieved two partial responses in 47 patients and 16 had disease stabilization. ${ }^{31}$ Phase I studies using the drug in combination with other chemotherapeutic agents indicated that the combinations were generally well tolerated. In a Phase I trial in solid tumors, in which tivantinib was given in combination with gemcitabine, three partial responses were observed in patients with NSCLC. ${ }^{32} \mathrm{Six}$ of eight patients with advanced NSCLC achieved disease stabilization in a Phase I study combining tivantinib and the EGFR inhibitor, erlotinib. ${ }^{33}$ Pharmacodynamic investigations performed in Phase I studies indicated a drop in intratumoral c-Met levels and a concomitant decrease in circulating tumor cells $(>30 \%)$ and endothelial cells (up to $100 \%){ }^{30}$

\section{Efficacy studies}

In a randomized Phase II trial comparing tivantinib plus erlotinib versus placebo plus erlotinib, tivantinib was given orally at a dose of $360 \mathrm{mg}$ twice daily. There were 84 patients in the tivantinib plus erlotinib arm and 83 in the placebo plus erlotinib arm. ${ }^{34}$ Overall and progression-free survival rates were slightly (albeit not significantly) in favor of tivantinib plus erlotinib, with a median overall survival of 8.5 months for tivantinib plus erlotinib and 6.9 for placebo plus erlotinib. All patients entering the study had, when feasible, molecular analysis including evaluation of EGFR and $K-R A S$ mutational status and Met gene copy number. Subgroup analysis according to molecular and histological characteristics indicated that patients with nonsquamous histology showed a trend in favor of tivantinib plus erlotinib in both overall and progression-free survival. A benefit for tivantinib plus erlotinib was also found for patients with wild-type $E G F R$, mutated $K-R A S$, and Met gene copy number greater than $2^{34}$ (see Figure 3). Further molecular analysis using immunohistochemistry to detect Met expression, conducted in the same samples, indicated that NSCLC with nonsquamous histology expressed higher levels of Met than squamous ones. ${ }^{35}$ Progression-free and overall survival rates were better in the tivantinib plus erlotinib arm in patients with high Met expression. It is of note that tivantinib plus erlotinib and placebo plus erlotinib gave similar results for overall and progression-free survival in the Met positive population.

Interestingly, patients in the tivantinib plus erlotinib arm had a significant delay in the development of new metastases, which was again more pronounced in the nonsquamous 


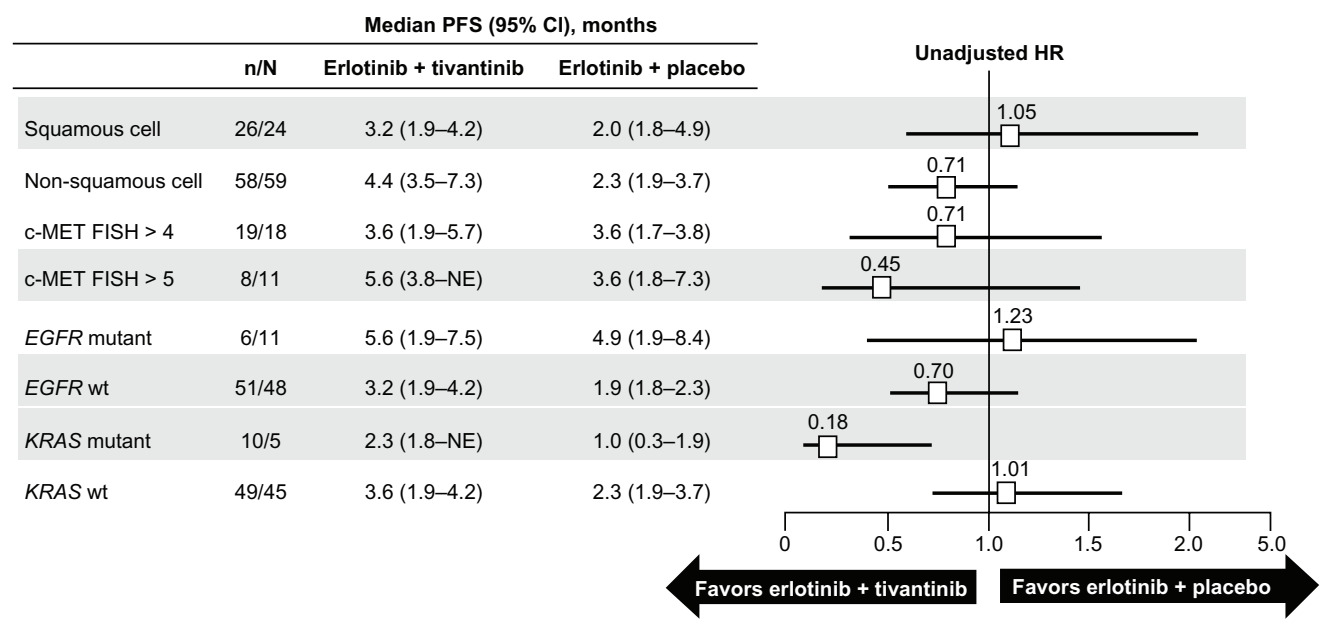

Figure 3 Cox proportional hazard ratio analysis of median progression-free survival by patient subgroup.

Reprinted from Scagliotti GV, Novello S, Schiller JH, et al. Rationale and design of MARQUEE: A Phase III, randomized, double-blind study of tivantinib plus erlotinib versus placebo plus erlotinib in previously treated patients with locally advanced or metastatic, nonsquamous, non-small-cell lung cancer. Clin Lung Cancer. 20I2; I3(5):39I-395, with permission from Elsevier. ${ }^{37}$

Abbreviations: $\mathrm{Cl}$, confidence interval; FISH, fluorescence in situ hybridization; HR, hazards ratio; PFS, progression-free survival.

population. ${ }^{34}$ This clinical evidence is in line with the preclinical antimetastatic activity reported for tivantinib in human models of metastasis developing in immunodeficient mice and suggests a possible additional role for tivantinib for preventing metastasis in early-stage malignancies. ${ }^{21,28}$

A randomized Phase II study is currently under way comparing erlotinib plus tivantinib versus single agent chemotherapy (gemcitabine, pemetrexed, or docetaxel) in previously treated, advanced, or metastatic patients with NSCLC and mutated $K-R A S$. The primary endpoint is progression-free survival, while secondary endpoints are overall survival, objective response rate in the entire population, and objective response rate "among subjects randomly assigned to chemotherapy who cross over following disease progression to receive erlotinib plus tivantinib and who are evaluable for a post-progression scan on crossover therapy" (clinical trial identifier NCT01395758).

Based on the encouraging Phase II results in NSCLC, a randomized, double-blind, Phase III trial (MARQUEE) is in progress in patients with advanced or metastatic nonsquamous NSCLC. ${ }^{36,37}$ The study has overall survival as its primary endpoint, and progression-free survival and overall survival in molecularly defined subgroups as secondary endpoints. All patients entering the study are molecularly profiled and evaluated for $E G F R$ and $K-R A S$ mutational status and for Met amplification and/or expression by immunohistochemistry. Additional planned analysis includes serum circulating hepatocyte growth factor (the ligand of Met) levels. The study is designed to detect a 33\% improvement in overall survival, requiring approximately 1000 patients to be enrolled. This number has been reached and the final results are expected in early 2013.

However, a recent press release announced that an independent data monitoring committee recommended the trial should be stopped early following a planned interim analysis because it would not meet the primary endpoint of improved overall survival. Although the interim analysis showed a statistically significant improvement in progression-free survival, this benefit did not translate into a benefit in overall survival. No safety concerns were identified by the data monitoring committee. It will be important to identify in which subgroups the drug had any effect.

\section{Long-term safety and tolerability issues}

Cumulative data (including for those reported in patients with NSCLC) from trials incorporating tivantinib as a single agent indicate that the drug is well tolerated. The most common drug-related toxicities are myelotoxicity, including neutropenia and febrile neutropenia, leucopenia, and thrombocytopenia. Fatigue and gastrointestinal toxicities have also been reported.

The combination of tivantinib with erlotinib in patients with NSCLC did not produce any additional toxicities (compared with erlotinib alone). ${ }^{33,34}$ Similarly, the combination of tivantinib and gemcitabine was generally well tolerated. ${ }^{32}$ Interim analysis of the large (more than 1000 patients enrolled) controlled randomized Phase III trial comparing erlotinib versus erlotinib plus tivantinib did not bring to light any safety concerns, in agreement with the data obtained in early studies using this combination. 


\section{Patient-focused perspectives}

In clinical trials, tivantinib in association with erlotinib showed superiority over erlotinib plus placebo. An interesting result from these randomized trials is that the combination of tivantinib plus erlotinib significantly delayed the development of new metastatic disease compared with erlotinib plus placebo. This delay was even more pronounced in cases with nonsquamous histology. Although this was not a classical clinical trial endpoint, it could have significant clinical implications, particularly with regard to patient quality of life.

\section{Conclusion}

Tivantinib has yielded interesting and promising results in advanced NSCLC. The encouraging results obtained with the monoclonal antibody (onartuzumab) targeting Met, in combination with erlotinib ${ }^{38}$ clearly indicate that the strategy of targeting Met in NSCLC is worth pursuing further. Tivantinib is well tolerated either as a single agent or in combination with other agents. This is an additional advantage for patients with advanced NSCLC. The Phase III trials will help to clarify whether subgroups of patients (based on histology, presence of mutations in $K-R A S$ or $E G F R$ ) could particularly benefit from this drug. The indication that Met expression can help in defining patients more likely to respond to tivantinib is an additional advantage when biological material is available for evaluation. Nevertheless, the results obtained so far clearly indicate that tivantinib has no detrimental effects, even in the absence of Met overexpression. This, together with the evidence that tivantinib retains its activity in patients with $K-R A S$ mutations (often inducing resistance to chemotherapy and targeted therapies) constitutes an additional advantage for the use of this drug in NSCLC. The sarcomatoid histotype could also be an interesting area for this drug, in considering its elevated Met expression. ${ }^{39}$ Met plays an important role in metastatic progression. The initial results obtained in advanced NSCLC showing that tivantinib can delay the onset of new metastatic disease are very encouraging. Even if not yet formally proven, addition of tivantinib to other therapies, not necessarily containing erlotinib, should give similar results, thus extending its possible use in combination with other drugs. The results in other malignancies where tivantinib has been combined with other drugs support the good combinability and efficacy of this agent.

\section{Acknowledgment}

The authors wish to thank JD Baggott who kindly edited the manuscript.

\section{Disclosure}

The authors report no conflicts of interest in this work.

\section{References}

1. Ferlay J, Shin HR, Bray F, Forman D, Mathers C, Parkin DM. Estimates of worldwide burden of cancer in 2008: GLOBOCAN 2008. Int $J$ Cancer. 2010;127(12):2893-2917.

2. World Health Organization. International Agency for Research on Cancer. World Cancer Report. Lyon, France: IARC Press; 2008.

3. Scagliotti GV, Parikh P, von Pawel J, et al. Phase III study comparing cisplatin plus gemcitabine with cisplatin plus pemetrexed in chemotherapy-naive patients with advanced-stage non-small-cell lung cancer. J Clin Oncol. 2008;26(21):3543-3551.

4. Maemondo M, Inoue A, Kobayashi K, et al. Gefitinib or chemotherapy for non-small-cell lung cancer with mutated EGFR. $N$ Engl J Med. 2010;362(25):2380-2388

5. Mok TS, Wu YL, Thongprasert S, et al. Gefitinib or carboplatinpaclitaxel in pulmonary adenocarcinoma. NEngl J Med. 2009;361(10) 947-957.

6. Shepherd FA, Rodrigues Pereira J, Ciuleanu T, et al. Erlotinib in previously treated non-small-cell lung cancer. $N$ Engl J Med. 2005;353(2): $123-132$.

7. Zhou C, Wu YL, Chen G, et al. Erlotinib versus chemotherapy as first-line treatment for patients with advanced EGFR mutation-positive non-small-cell lung cancer (OPTIMAL, CTONG-0802): a multicentre, open-label, randomised, phase 3 study. Lancet Oncol. 2011;12(8): 735-742.

8. Yang JC-H, Schuler MH, Yamamoto N, et al. LUX-Lung 3: a randomized, open-label, phase III study of afatinib versus pemetrexed and cisplatin as first-line treatment for patients with advanced adenocarcinoma of the lung harboring EGFR-activating mutations. ASCO Meeting Abstracts. 2012;30(Suppl 15):LBA7500.

9. Kwak EL, Bang Y-J, Camidge DR, et al. Anaplastic lymphoma kinase inhibition in non-small-cell lung cancer. N Engl J Med. 2010;363(18): 1693-1703.

10. Garassino MC, Martelli O, Bettini A, et al. TAILOR: Phase III trial comparing erlotinib with docetaxel in the second-line treatment of patients with NSCLC with wild-type (wt) EGFR. ASCO Meeting Abstracts. 2012;30(Suppl 15):LBA7501.

11. Choi YL, Soda M, Yamashita Y, et al. EML4-ALK mutations in lung cancer that confer resistance to ALK inhibitors. $N$ Engl J Med. 2010;363(18):1734-1739.

12. Kobayashi S, Boggon TJ, Dayaram T, et al. EGFR mutation and resistance of non-small-cell lung cancer to gefitinib. $N$ Engl J Med. 2005;352(8):786-792.

13. Pao W, Miller VA, Politi KA, et al. Acquired resistance of lung adenocarcinomas to gefitinib or erlotinib is associated with a second mutation in the EGFR kinase domain. PLoS Med. 2005;2(3):e73.

14. Sasaki T, Koivunen J, Ogino A, et al. A novel ALK secondary mutation and EGFR signaling cause resistance to ALK kinase inhibitors. Cancer Res. 2011;71(18):6051-6060.

15. Bean J, Brennan C, Shih JY, et al. MET amplification occurs with or without T790M mutations in EGFR mutant lung tumors with acquired resistance to gefitinib or erlotinib. Proc Natl Acad Sci USA. 2007;104(52):20932-20937

16. Engelman JA, Zejnullahu K, Mitsudomi T, et al. MET amplification leads to gefitinib resistance in lung cancer by activating ERBB3 signaling. Science. 2007;316(5827):1039-1043.

17. Kris MG, Johnson BE, Kwiatkowski DJ, et al. Identification of driver mutations in tumor specimens from 1,000 patients with lung adenocarcinoma: the NCI's Lung Cancer Mutation Consortium (LCMC). ASCO Meeting Abstracts. 2011;29(Suppl 18):CRA7506.

18. Trusolino L, Bertotti A, Comoglio PM. MET signalling: principles and functions in development, organ regeneration and cancer. Nat Rev Mol Cell Biol. 2010;11(12):834-848. 
19. Ma PC, Tretiakova MS, MacKinnon AC, et al. Expression and mutational analysis of MET in human solid cancers. Genes Chromosomes Cancer. 2008;47(12):1025-1037.

20. Ma PC, Jagadeeswaran R, Jagadeesh S, et al. Functional expression and mutations of c-Met and its therapeutic inhibition with SU11274 and small interfering RNA in non-small cell lung cancer. Cancer Res. 2005;65(4):1479-1488.

21. Munshi N, Jeay S, Li Y, et al. ARQ 197, a novel and selective inhibitor of the human c-Met receptor tyrosine kinase with antitumor activity. Mol Cancer Ther. 2010;9(6):1544-1553.

22. Stella GM, Benvenuti S, Comoglio PM. Targeting the MET oncogene in cancer and metastases. Expert Opin Investig Drugs. 2010;19(11): 1381-1394.

23. Jin H, Yang R, Zheng Z, et al. MetMAb, the one-armed 5D5 anti-c-Met antibody, inhibits orthotopic pancreatic tumor growth and improves survival. Cancer Res. 2008;68(11):4360-4368.

24. Eathiraj S, Palma R, Volckova E, et al. Discovery of a novel mode of protein kinase inhibition characterized by the mechanism of inhibition of human mesenchymal-epithelial transition factor (c-Met) protein autophosphorylation by ARQ 197. J Biol Chem. 2011;286(23): 20666-20676.

25. Mao C, Qiu L-X, Liao R-Y, et al. KRAS mutations and resistance to EGFR-TKIs treatment in patients with non-small cell lung cancer: a meta-analysis of 22 studies. Lung Cancer. 2010;69(3):272-278.

26. Marabese M, Rulli E, Bettini A, et al. KRAS mutational status impact progression-free survival of patients treated with platinum-based chemotherapy in NSCLC. Mol Cancer Ther. 2011;10 Suppl 1:B77.

27. Metro G, Chiari R, Duranti S, et al. Impact of specific mutant KRAS on clinical outcome of EGFR-TKI-treated advanced non-small cell lung cancer patients with an EGFR wild type genotype. Lung Cancer. 2012;78(1):81-86.

28. Previdi S, Abbadessa G, Dalo F, France DS, Broggini M. Breast cancerderived bone metastasis can be effectively reduced through specific c-Met inhibitor tivantinib (ARQ 197) and shRNA c-Met knockdown. Mol Cancer Ther. 2012;11(1):214-223.

29. Savage R, Zhong C, Hall T, et al. In vitro ADME properties of ARQ-197. Comparison to in vivo data. Poster 1291 presented at the 99th American Association for Cancer Research Annual Meeting, Philadelphia, PA, April 12-16, 2009.

30. Yap TA, Olmos D, Brunetto AT, et al. Phase I trial of a selective c-Met inhibitor ARQ 197 incorporating proof of mechanism pharmacodynamic studies. J Clin Oncol. 2011;29(10):1271-1279.
31. Yamamoto N, Nishina T, Sugio K, et al. Two patients with NSCLC achieved PR by ARQ 197 monotherapy. Final results of the first Asian phase I trial of selective c-Met inhibitor ARQ 197. J Thorac Oncol. 2011;6(6):S1170.

32. Camacho LH, Bendell JC, Pant S, et al. Phase 1B results of c-Met inhibitor tivantinib (ARQ 197) in combination with gemcitabine (GEM) in a cohort of patients (PTS) with advanced thoracic tumors. J Thorac Oncol. 2011;6(6):S1265.

33. Goldman JW, Laux I, Chai F, et al. Phase 1 dose-escalation trial evaluating the combination of the selective MET (mesenchymal-epithelial transition factor) inhibitor tivantinib (ARQ 197) plus erlotinib. Cancer. 2012;118(23):5903-5911.

34. Sequist LV, Von Pawel J, Garmey EG, et al. Randomized phase II study of erlotinib plus tivantinib versus erlotinib plus placebo in previously treated non-small-cell lung cancer. J Clin Oncol. 2011;29(24): 3307-3315.

35. Rodig SJ, Sequist L, Schiller JH, et al. An exploratory biomarker analysis evaluating the effect of the c-Met inhibitor tivantinib (ARQ 197) and erlotinib in patients with NSCLC in a randomized, double-blinded phase 2 study. Paper presented at the 102nd Annual Meeting of the American Association for Cancer Research, Chicago, IL, March 31-April 4, 2012.

36. Sandler A, Schiller JH, Hirsh V, et al. A phase III, randomized, doubleblind, placebo-controlled study of erlotinib plus ARQ 197 versus erlotinib plus placebo in previously treated subjects with locally advanced or metastatic, nonsquamous, non-small cell lung cancer (NSCLC). ASCO Meeting Abstracts. 2011;29(Suppl 15):TPS217.

37. Scagliotti GV, Novello S, Schiller JH, et al. Rationale and design of MARQUEE: A Phase III, randomized, double-blind study of tivantinib plus erlotinib versus placebo plus erlotinib in previously treated patients with locally advanced or metastatic, nonsquamous, non-small-cell lung cancer. Clin Lung Cancer. 2012;13(5):391-395.

38. Fasolo A, Sessa C, Gianni L, Broggini M. Seminars in clinical pharmacology: an introduction to MET inhibitors for the medical oncologist. Ann Oncol. 2013;24(1):14-20.

39. Pelosi G, Gasparini P, Cavazza A, et al. Multiparametric molecular characterization of pulmonary sarcomatoid carcinoma reveals a nonrandom amplification of anaplastic lymphoma kinase (ALK) gene. Lung Cancer. 2012;77(3):507-514.
Cancer Management and Research

\section{Publish your work in this journal}

Cancer Management and Research is an international, peer-reviewed open access journal focusing on cancer research and the optimal use of preventative and integrated treatment interventions to achieve improved outcomes, enhanced survival and quality of life for the cancer patient. The journal welcomes original research, clinical \& epidemiological studies, reviews \& evaluations, guidelines, expert opinion \& commentary, case reports \& extended reports. The manuscript management system is completely online and includes a very quick and fair peerreview system, which is all easy to use. Visit http://www.dovepress.com/ testimonials.php to read real quotes from published authors. 